\title{
ANÁLISIS Y BALANCE SOBRE EL DESARROLLO DE LA FUNCIÓN DIRECTIVA EN EL PERÚ: EL CASO DEL CUERPO DE GERENTES PÚBLICOS*
}

\author{
Rafael Martínez Puón \\ Universidad Nacional Autónoma de México \\ rafael.martinez@ine.mx
}

\section{RESUMEN}

El artículo estudia el desarrollo de la función directiva en la administración pública peruana. Se analiza el caso de los directivos regulados por Ley de Servicio Civil de 2013, los tipos de directivos públicos establecidos por la ley, las diferentes modalidades que se establecen para el acceso a los puestos. El artículo también se centra en el Cuerpo de Gerentes Públicos. Específicamente, se describen los procesos que integran su modelo de gestión y los perfiles de competencias diseñados SERVIR, la agencia a cargo del cuerpo. Con posterioridad se describen los resultados obtenidos en la implementación del cuerpo. Finalmente, se identifican las principales fortalezas y áreas de oportunidad para el desarrollo del Cuerpo de Gerentes Públicos.

Palabras clave: Función directiva, Cuerpo de Gerentes Públicos, Servicio civil, Perú.

* El autor quiere agradecer el apoyo de Andrés Angulo, Directivo de SERVIR por el apoyo en la precisión de algunos puntos expuestos en una primera versión de este documento, a partir de entrevista realizada el 10 de julio de 2015, y a Alejandro Porras Calderón, por su labor en la recopilación de la información e investigación. 


\title{
ANALYSIS AND BALANCE ON THE DEVELOPMENT OF THE DIRECTIVE FUNCTION IN PERU: THE CASE OF THE CUERPO DE GERENTES PÚBLICOS
}

\begin{abstract}
This article examines the development of the managerial function in the Peruvian Public Administration. The case of managers under the 2013 Civil Service Act is analyzed, along with the types of public managers established by the Act, and the different modalities established for access to the posts. In second place, the article focuses on the Cuerpo de Gerentes Públicos (Public Managers Cadre). Specifically, it goes through the processes that make up its management model and the competency profiles designed by SERVIR (the agency in charge of the Cuerpo). Subsequently the results in the implementation of this Civil Service are described. Finally, the main strengths and areas of opportunity for the development of the Cuerpo de Gerentes Públicos are identified.
\end{abstract}

Keywords: Directive function, Public Managers Cadre, Civil Service, Peru. 


\section{INTRODUCCIÓN}

Hablar de los esfuerzos de profesionalización ${ }^{1}$ de las funciones públicas latinoamericanas, sigue siendo un tema más de excepción que de normalidad burocrática. Y lo decimos de esta manera, porque si bien en los últimos años a partir, de la ahora fuente obligada sobre este asunto, como lo es el documento Al servicio del ciudadano: Una década de reformas del servicio civil en América Latina (2004-2013), auspiciado por la División de Capacidad Institucional del Estado del Banco Interamericano de Desarrollo, en el que se reconoce un esfuerzo importante de todos de los países de la región en el impulso y consolidación de este tema. Desde nuestro punto de vista, se considera que la profesionalización sigue siendo un bien escaso, con experiencias consolidadas mínimas, con esfuerzos discontinuos en la mayoría de los países (sobre todo en los cambios de gobiernos y por la llegada de nuevos partidos), sino es que en algunos casos, la situación prevaleciente es la existencia de leyes muy bien diseñadas, pero no así implementadas.

Sin embargo, lo que llama poderosamente la atención es que, a pesar de todo lo mencionado, cada país no deja de emitir iniciativas y desarrollar esfuerzos, a partir de sus propias circunstancias tanto políticas como administrativas. Y sobre esa base, incluso adoptar todo lo expresado en las tendencias en materia de gestión de recursos humanos. Al respecto, si bien es cierto que la figura emblemática lo siguen siendo los servicios civiles o profesionales de carrera ${ }^{2}$, hay un interés por la posibilidad de segmentar la profesionalización hacia cierto público de servidores o funcionarios públicos. En esta idea, está un segmento muy importante compuesto por los denominados directivos públicos. Lo que ha venido a denominarse como la profesionalización de la función directiva o lo que se identifica, en algunos países, como senior civil service o los executive civil service (países anglosajones), función directiva profesional o gerentes públicos (países de Europa Continental o América Latina).

En los últimos quince años, Perú junto con otros países, como Chile, Brasil, República Dominicana, Colombia (Figueroa 2002), se han hecho a la tarea de impulsar la profesionalización de este segmento, a la par del

1 Entiéndase como la capacidad que tienen las organizaciones de dotar a su personal de las capacidades necesarias para que en aras de su desarrollo individual, este contribuya en el logro de los objetivos de las mismas.

2 Como aquel sistema de empleo en el que las personas ingresan a la función pública por sus méritos y en igualdad de condiciones. 
desarrollo y consolidación de sus servicios civiles, por lo que todavía las experiencias en este rubro, son aún más escasas. Concretamente, habría que destacar el caso del Cuerpo de los Gerentes Públicos como asunto a destacar en contraparte, con la figura que existe a nivel del Ley del Servicio Civil (2013) que es la de directivos públicos.

El propósito de este artículo es explicar el esfuerzo en la profesionalización de directivos denominado Cuerpo de Gerentes Públicos, aunque esto no excluye la exposición con respecto a los directivos públicos regulados por la citada Ley del Servicio Civil. Esta aclaración es importante hacerla, porque estamos hablando de sistemas paralelos. Todo parece indicar, que uno mejor que otro, el de gerentes sobre directivos, y que por lo visto, son contrarios en cuanto a su desarrollo. El artículo da cuenta de su diseño y algunos de sus resultados, así como algunas recomendaciones.

En el texto se hace una revisión y análisis conceptual de la evolución que ha tenido el desarrollo de la función directiva en el Perú, cuyo contenido inicia con la explicación de los elementos teóricos de la función directiva y el papel de los directivos públicos, para después describir los factores institucionales que detonaron el impulso de la función directiva en ese país que se concretó con la expedición de la Ley del Servicio Civil de 2013, los tipos de directivos públicos establecidos por la norma, las diferentes modalidades que se establecen para el acceso a los puestos, así como los principales problemas que se observan para el avance de la función directiva. Posteriormente, se aborda la forma cómo se define al Cuerpo de Gerentes Públicos, se describen los procesos que integran su modelo de gestión, los perfiles de competencias que deben tener de acuerdo a lo que determina la Autoridad Nacional del Servicio Civil (SERVIR), la entidad rectora responsable de este cuerpo; se presentan algunos resultados obtenidos en la implementación de ese cuerpo; se identifican las principales fortalezas y áreas de oportunidad para el desarrollo del Cuerpo de Gerentes Públicos en el Perú; y por último se presentan las conclusiones.

\section{APROXIMACIONES TEÓRICAS Y DEFINICIÓN DE LA DIRECCIÓN PÚBLICA Y LOS DIRECTIVOS PÚBLICOS}

Si bien, a lo largo del escrito se hará alusión al término gerentes y directivos, para efectos conceptuales, el término que se adoptará es el de dirección, función directiva o dirección pública. En ese sentido, hablar de directivos, necesariamente, se tiene que hacer alusión a estudios que ya resultan ser clásicos, como los de Stewart (1988), Likert (1961), Sayles (1964), Kotter (1983) y, principalmente, los de Mintzberg (1983, 1996, 2005, 2006), cuyo desarrollo ha sido tanto en el ámbito privado como en el ámbito 
público, así como los trabajos de Laufer y Burluad (1989), O estudios muy particulares en algunas funciones públicas como los de Dauphinais y Price (1998) o de la propia Organización para la Cooperación y el Desarrollo Económicos (OCDE) (1999). En el ámbito latinoamericano destaca el trabajo de Kliksberg (1983). Y en cuanto a definiciones, señalar las siguientes:

La dirección no es un simple componente de la gestión, tiene que ver con múltiples talentos, entre los que deben figurar la integridad, la intuición, la capacidad de inspirar a los demás, una profunda conciencia de sí, el valor de innovar y un discernimiento rápido e infalible. La dirección supone un espíritu inquebrantable, un espíritu visionario, y un real afecto por las personas a las que se dirige" (Brosnahan 2000).

La dirección como la movilización de personas para hacer frente a realidades problemáticas, afrontarlas y resolverlas" (Heifetz 1994)

La dirección como el proceso de persuasión o el ejemplo por el que el individuo (o un equipo) conduce a un grupo a perseguir objetivos defendidos por los directivos o compartidos por el directivo y sus seguidores (Gardner 1990).

Como se puede observar, el tema nos abre una serie de posibilidades sobre cómo entender y abordar un asunto de esta naturaleza.

Ahora bien, es importante señalar que si bien la atención sobre el desarrollo e institucionalización de la función directiva tuvo como primer escenario a las organizaciones privadas (como habitualmente ha acontecido), en las últimas décadas, sobre todo a finales de los años setenta y no se diga últimamente (v véase The Governance Report (2014), realizado por Martin Lodge y Kai Wegrich) ha cobrado una importancia relevante a nivel de organizaciones públicas. Primeramente, a partir de la construcción de lo que se conoce como servicios civiles senior, al respecto hay muchos casos emblemáticos (Estados Unidos, Inglaterra, Holanda, Suecia, entre otros. Véase Martínez Puón 2011), pero además como una tendencia que se ha extendido a muchos países, hasta alcanzar a los países latinoamericanos, aunque, como se comentó en la introducción, con experiencias escasas. Y nos parece que en gran parte, por la ausencia de servicios profesionales de carrera consolidados.

Según la OCDE (1999: 9), si se tuviera que definir una carrera para directivos públicos, se diría que es una estructura y un sistema reconocido de gestión para altas posiciones no políticas (llámese para funciones de 
directivos públicos) en el gobierno. Es un sistema de servicio civil que entrena y forma directivos para el desempeńo de funciones ad-hoc. Es un servicio que, principalmente, es gestionado a través de instituciones y procesos apropiados para proveer estabilidad y profesionalismo a este grupo de altos directivos, pero con la necesaria flexibilidad en la composición de los gobiernos.

De esta definición se tienen que destacar los siguientes elementos (OCDE 2008):

- Las posiciones más altas en el gobierno. Los puestos ocupados por el servicio civil senior son los más altos en jerarquía (en departamentos centrales y agencias), normalmente se sitúan justo debajo de los nombramientos políticos que se dan mediante procedimientos no competitivos. El número de niveles que comprende el servicio civil senior depende de la definición usada por cada país. Los puestos más altos del gobierno pueden definirse por medio de un rango específico de posiciones (secretarías generales o directores generales), estableciendo un grado o escala personal que sean atribuibles a la persona y al puesto o mediante un grupo salarial.

- Nombramientos competitivos. El nombramiento abierto significa que hay una adecuada convocatoria para candidatos internos y externos, y basado en el mérito, las capacidades, experiencia, desempeño de los candidatos.

- Instituciones y procesos adecuados de gestión de recursos humanos. Nombramiento, remociones, promociones, evaluación del desempeño, salarios y demás, se determinan por procesos en los que se introducen mecanismos de equilibrio de poder. Este equilibrio es necesario para asegurar la competencia abierta y evitar la interferencia política. En algunos casos, hay una comisión que hace los nombramientos dentro del departamento, la cual evalúa y clasifica a los candidatos. Este trabajo puede ser verificado por un cuerpo independiente, o el propio cuerpo independiente puede organizar todo el proceso de nombramiento o parte de él. En estos ejemplos, las instituciones y los procedimientos otorgan una competencia real al reclutamiento. En otros países, el candidato es seleccionado por una vacante superior. En estos casos, los criterios de competencia a menudo se anulan en la práctica, por ejemplo, simulan la descripción del perfil del puesto para anunciarlo en la gaceta oficial para potencial preselección del candidato, incluso publican antes la vacante. No existe una definición universal de instituciones y procesos adecuados de gestión de recursos humanos, ya que las instituciones tienen contextos específicos.

- Funciones. Los servidores civiles senior pueden desempeñar una 
o varias funciones esenciales: 1) asesoría política, analizando e informando sobre contenidos políticos a ministros, normalmente sin incluir la opinión de partidos políticos, que podría ser el trabajo de los asesores o nombramientos políticos; 2) eficacia operativa, administrando recursos para alcanzar los resultados esperados de los programas y servicios; 3) eficacia en servicios corporativos, gestión de recursos financieros, personal, tecnología e infraestructura.

- Separación formal de un grupo definido. La separación significa que el personal perteneciente al servicio civil senior, tiene diferentes reglas y planes del resto del servicio civil. Aquellos planes diferentes podrían ser estructura salarial, contratación, nombramiento, acuerdos de desempeño y evaluación, sistema de equilibrio de poderes, entre otros.

- Flexibilidad, respetando los procesos. La flexibilidad, quizás, necesite homogeneizar los procesos competitivos en el nombramiento, para la correcta relación entre servidores civiles senior y el ministro (o nombramientos políticos). Podría acompañarse de procesos adecuados, cuando, por ejemplo, los ministros tienen la oportunidad de aceptar o rechazar un nombramiento de una comisión autónoma, o acceden a evaluación independiente cuando hay un desempeño poco confiable o inadecuado, pueden destituir a un servidor civil senior. En estos casos, deberían de respetarse los procesos para evitar la arbitrariedad de los políticos.

- Dirección centralizada. Los servicios civiles senior deben estar centralizados o monitoreados para prevenir la interferencia política que se da en el nivel ministerial individual, especialmente en promoción, descenso y salarios basados en el desempeño.

En la misma línea de pensamiento, la Carta Iberoamericana de la Función Pública señala:

[...] los directivos públicos corresponden a aquel segmento de cargos o dirección inmediatamente subordinado al nivel político de los gobiernos, cuya función es dirigir, bajo la orientación estratégica y el control de aquel, las estructuras y procesos mediante los cuales se implementan las políticas públicas y se producen y proveen los servicios públicos. Se trata de una función diferenciada tanto de la política como de los profesionales de las políticas públicas que integran la función pública ordinaria. La adecuada definición y consolidación de una dirección pública profesional son básicas para un correcto diseño institucional de los sistemas públicos contemporáneos (CLAD-UN 2004). 
En efecto, lo que habría que enfatizar es esta diferenciación de la función directiva (a la usanza de lo que prevalece en los servicios profesionales senior) con respecto a la función pública profesional ordinaria, que va desde la existencia de normas y procesos distintos para cada segmento, con excepciones en las que un mismo ordenamiento contiene y regula a ambos, y que apuntan a centrar la atención en funciones públicas con especializaciones diferenciadas.

Nos parece que, no obstante los avances conseguidos, se siguen reconociendo déficits de institucionalización y de estudios por realizar; este es un tema que está, seguramente como otros tantos, sujeto todavía de profundas revisiones que reta a nuevos descubrimientos. El caso latinoamericano (Figueroa 2002) es un campo sumamente fértil para explorar estas nuevas líneas de estudio. De ahí que se destaque lo que se ha realizado en el Perú, que se considera que es una propuesta atractiva e innovadora, que bien puede ser de utilidad en los estudios comparados.

\section{¿CÓMO SURGE LA NECESIDAD DE PROPULSAR LA FUNCIÓN DIRECTIVA EN PERÚ?}

Como ya se señaló, en sus comienzos el Banco Interamericano de Desarrollo publicó en 2004 un diagnóstico de los servicios civiles en América Latina, en el que ubicó a Perú en el lugar 17 sobre 21 países en el ranking ${ }^{3}$ (Iacoviello 2011), evidenciando una débil institucionalidad. Lo que hay que destacar de este diagnóstico es que mide varios aspectos a través de diversos índices (eficiencia, mérito, consistencia estructural, capacidad funcional y capacidad integradora). No suficiente con esto, estos índices se dividen a su vez en subíndices. El índice de consistencia tiene tres subíndices y uno de ellos tiene que ver con el grado de desarrollo de la función directiva, y sobre este rubro, Perú ocupo el puesto 14 (Corrales 2012). En el año 2008, el gobierno del Presidente Alan García impulsó una sucesión de cinco decretos que dieron lugar a la Reforma de su Servicio Civil, creando a través del Decreto N. ${ }^{\circ} 1.023$ la Autoridad Nacional del Servicio Civil (SERVIR), y mediante el Decreto N. ${ }^{\circ} 1.024$ el Cuerpo de Gerentes Públicos.

Según Iacovello (2011), SERVIR surgió ante la falta de un órgano rector que normara y ordenara el sistema de recursos humanos. Es importante destacar que en el Decreto Legislativo se estableció que SERVIR sería un organismo técnico especializado, adscrito a la Presidencia del Consejo de Ministros, que tenía como finalidad contribuir a la mejora continua de

3 Este informe se actualizó en 2013. 
la administración del Estado a través del fortalecimiento del servicio civil (Decreto N. ${ }^{\circ} 1.023$, artículos 1 y 6). En palabras de Alza Barco (2012), se le ordenó a SERVIR la tarea de crear un Cuerpo de Gerentes Públicos que le permitiera contar con un sistema de puestos directivos meritocrático y fuertemente orientado a metas, que condujera todo el proceso de gestión de la administración pública.

A este Cuerpo de Gerentes Públicos se le asignaron cuatro objetivos substanciales:

- Convocar profesionales capaces para altos puestos de dirección y gerencias de mando medio, a través de procesos transparentes y competitivos.

- Desarrollar capacidades de dirección y gerencia en la administración pública y asegurar su continuidad.

- Profesionalizar gradualmente los niveles más altos de la administración pública.

- Impulsar la reforma del Servicio Civil (Decreto N. ${ }^{\circ}$ 1.024, artículo 2).

En 2011, después de dos años de implantación de la reforma, el mismo equipo del Banco Interamericano de Desarrollo (BID) instrumentó una nueva medición sobre la situación que guardaba el servicio civil en Perú, encontrando avances significativos del $40 \%$ con respecto a profesionalización y mérito, mientras que $30 \%$ de mejora en consistencia estructural y capacidad funcional; esos resultados se atribuyeron, en gran parte, a la creación del Cuerpo de Gerentes Públicos y de SERVIR como entidad rectora (Iacoviello 2011).

Dos años después, a iniciativa del Poder Ejecutivo, el Congreso de la República aprobó la Ley del Servicio Civil (Ley N. ${ }^{\circ} 30.057$ ), en la que se estableció un régimen único y exclusivo para las personas que prestaban servicios en las entidades públicas del Estado, así como para aquellas personas que estaban encargados de su gestión (esto es, de los directivos públicos), del ejercicio de sus potestades y de la prestación de servicios a cargo de esas entidades (SERVIR 2015).

En el marco de profesionalizar el servicio civil en Perú, para entregar mejores servicios a la ciudadanía, las autoridades gubernamentales se fijaron el propósito de institucionalizar y profesionalizar la función directiva pública, basándose en tres principios fundamentales (SERVIR 2015):

- Creación de valor público.

- Responsabilidad de resultados. 
- Racionalidad económica

$-$

Lo que vale la pena, comentar aquí es que ambos sistemas, tanto el de la función directiva del servicio civil, como el de gerencia pública, se encuentran paralelamente en fase de desarrollo, como prácticas no necesariamente institucionalizadas sino como una especie de grandes laboratorios o pruebas piloto, hasta antes de la implementación de la Ley del Servicio Civil, prevista para seis años. Vayamos a la descripción de cada uno de ellos.

\section{¿DE QUÉ MANERA SE PUEDEN DEFINIR LOS DIRECTIVOS PÚBLICOS?}

Los directivos públicos se pueden definir como los servidores civiles que dirigen, bajo la orientación estratégica de los funcionarios públicos, las estructuras y procesos mediante los cuales se implementan las políticas públicas y se proveen bienes y servicios públicos (SERVIR 2015, basado en la Carta Iberoamericana de la Función Pública, artículo 53).

Se encuentran situados entre el grupo de funcionarios públicos (políticos y funcionarios de alta dirección de las entidades) y el grupo de profesionales o servidores de carrera (SERVIR 2015). 


\section{Gráfico 1: Fronteras del grupo de directivos públicos}
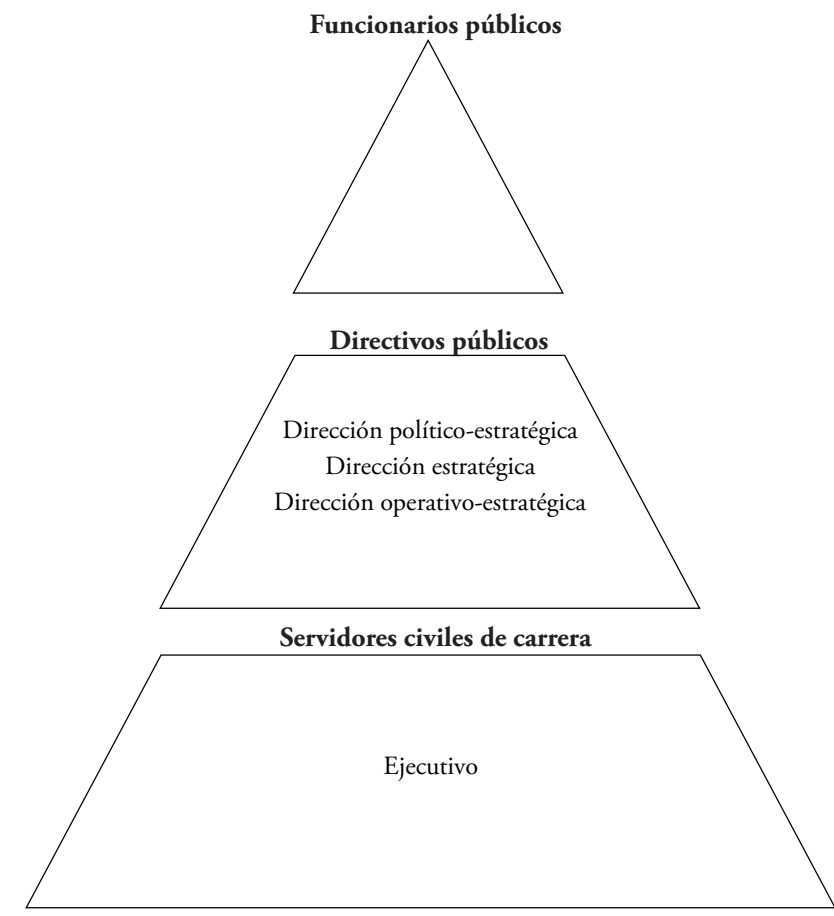

Servidores de actividades complementarias

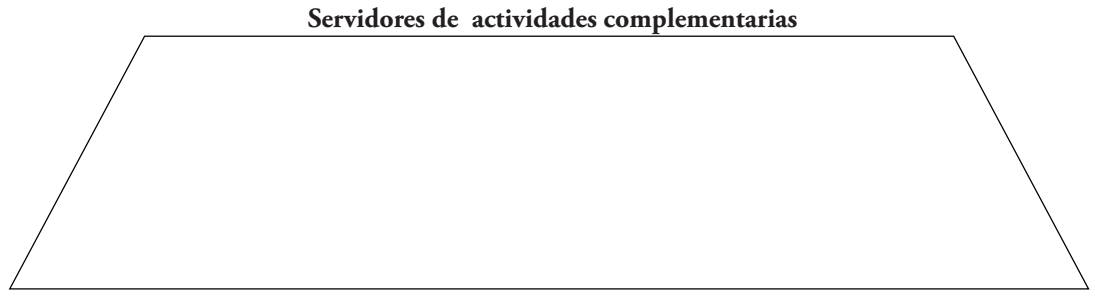

Fuente: SERVIR (2015: 45).

Es importante destacar que hasta que la Ley del Servicio Civil se implemente en todas las entidades públicas, no existen todavía los servidores públicos de carrera a que se refiere ese marco normativo. Existen todavía profesionales bajo diversas modalidades, unos del antiguo régimen de carrera, otros contratados bajo un régimen transitorio (contrato administrativo de servicios).

Se calcula que la composición del grupo de directivos públicos en el Estado ascienda a un número aproximado de 3.603 puestos, de los 6.039 puestos jefaturales identificados. La entidad rectora hizo una estimación por tipo de directivo, mismo que podría variar en la medida en que las entidades realicen su dimensionamiento de puestos directivos (SERVIR 2015). 


\section{Gráfico 2: Cantidad de directivos por tipo de directivo de gobierno nacional}

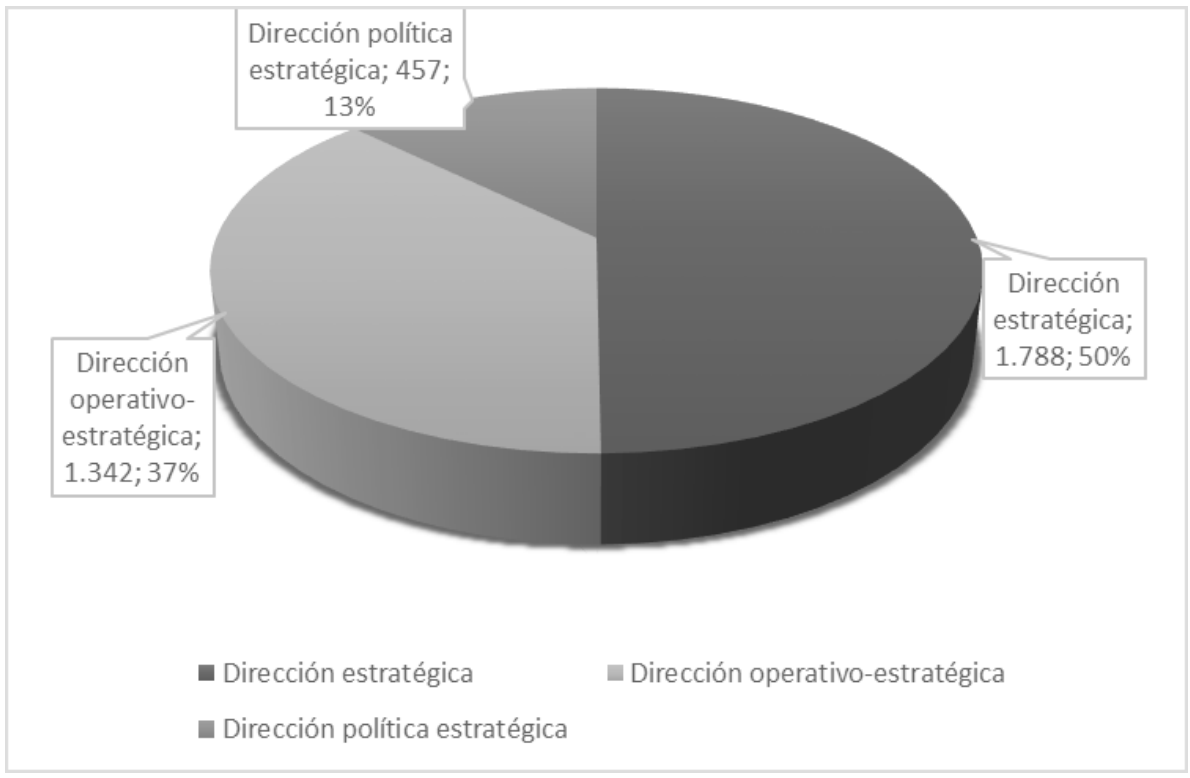

Fuente: SERVIR (2015: 49).

Está previsto que este cuerpo funcione de manera transitoria, sirviendo inicialmente a la puesta en marcha de la profesionalización del nivel directivo, para después de transcurridos los diez años el proceso de implementación de la reforma, integrarlo a lo que será la regulación general de los directivos públicos. De acuerdo con Corrales Ángulo (2010a), el propósito es que sirva para poner en marcha el proceso de profesionalización de la gerencia pública, para que luego pueda ser extendido hacia todos los demás directivos públicos.

\section{¿CUÁLES SON LOS TIPOS DE DIRECTIVOS PÚBLICOS?}

SERVIR (2015) establece tres modalidades de directivos: políticoestratégico, estratégicos y operativo-estratégico. En ese sentido, ¿Cuáles son sus ámbitos de la función directiva? (SERVIR 2015, basado en Moore):

- Los directivos político-estratégicos. Son los que identifican los tipos de actores presentes y buscan su apoyo a través de procesos formales, eligen vías adecuadas para la decisión, analizan escenarios políticos, aprovechan ventanas de oportunidad, gestionan la incertidumbre, entre otros.

- Los directivos estratégicos. Son los que adecuan los objetivos, 
estrategias y políticas definidas por las entidades a la generación de resultados. Implica la utilización de técnicas analíticas de planificación estratégica y de la habilidad de gestión de los directivos.

- Los directivos operativo-estratégicos. Son los que realizan el despliegue consciente y especializado de capacidades legales, financieras, materiales y humanas para obtener resultados concretos.

Esos tres tipos de directivos son considerados como los niveles de la rama directiva, siendo el énfasis mostrado en alguna o algunas esferas de gestión, las que determinan el tipo de dirección del puesto directivo (SERVIR 2015)

\section{¿CUÁLES SON LAS MODALIDADES PARA EL ACCESO A PUESTOS DEL GRUPO DE DIRECTIVOS PÚBLICOS Y CUÁL ES SU PERFIL DE COMPETENCIAS?}

SERVIR establece que, para acceder a un puesto de directivo público, es esencial que la entidad pública a la que pertenecen dichos puestos haya transitado al Régimen del Servicio Civil; una vez que se haya realizado ese proceso de tránsito, existen tres modalidades para ingresar a un puesto directivo: 1) por concurso de méritos; 2) por cumplimiento del perfil de puesto; y 3) mixta (SERVIR 2015). De acuerdo a la Ley del Servicio Civil, la selección de los directivos públicos los realiza cada entidad pública. Las modalidades consisten en:

- Ingreso por concurso de méritos. Es el ingreso al grupo de Directivos Públicos después de cumplir de forma satisfactoria todas las etapas del proceso de selección y resultar ganador del concurso público de méritos por el puesto directivo. Tiene dos variantes: 1) ingreso vertical. Se produce cuando un servidor público de carrera resulta ganador del concurso de méritos de un puesto directivo; 2) ingreso externo. Se genera cuando una persona natural, ajena o no al nuevo Régimen del Servicio Civil, resulta ganador del concurso.

- Ingreso por cumplimiento del perfil del puesto. Se producirá bajo tres supuestos: 1) ingreso horizontal. Es cuando un directivo público ingresa a otro puesto directivo del mismo tipo de dirección, por la causal de reubicación del puesto hasta que concluya el periodo para el que fue contratado; 2) ingreso por ser gerente público: es cuando un gerente público está asignado a un puesto directivo en una entidad pública que ha concluido su tránsito al nuevo régimen. La entidad pública puede incorporarlo como directivo bajo el Régimen del Servicio Civil sin necesidad de concurso; 3) puesto directivo ocupado por servidor de confianza. Es cuando un servidor de confianza cumple 
con el perfil de un puesto directivo y es designado para que ocupe el mismo.

- Modalidad mixta. La movilidad de un directivo público será parcial y no absoluta, así, un director operativo-estratégico dispone de movilidad (ingreso horizontal) únicamente a otros puestos de dirección operativa, salvo que lo haga por concurso abierto; un director estratégico dispondrá de movilidad a otros puestos de dirección operativo-estratégica y dirección estratégica; y un director político-estratégico gozará de movilidad a todos los puestos del segmento directivo; con la condición de cumplir con el perfil del puesto directivo requerido (SERVIR 2015).

Respecto a cuál es el perfil de competencias que deben tener, SERVIR establece para los directivos públicos un conjunto de competencias genéricas consistentes en:

- Liderazgo

- Articulación del entorno político

- Visión estratégica

- Capacidad de gestión

Asimismo, SERVIR determina tres competencias comunes a todos los servidores civiles y por ende también para los directivos públicos:

- Vocación de servicio

- Orientación a resultados

- Trabajo en equipo

\section{¿CUÁL ES LA PROBLEMÁTICA IDENTIFICADA EN LA FUNCIÓN DIRECTIVA?}

Históricamente el Estado peruano se ha caracterizado por tener un modelo de dirección pública politizado. Los directivos públicos han sido en su gran mayoría empleados de confianza de la alta dirección de las entidades públicas. Si bien es cierto, existe un margen racional para la contratación de empleados de confianza, un porcentaje muy alto para la confianza genera grandes márgenes de discrecionalidad que impiden la profesionalización de la función directiva en beneficio de los ciudadanos (SERVIR 2015). Aunque esto tampoco es exclusivo de la función directiva del Perú, la realidad es que tratándose este segmento es capturado fácilmente por los políticos en turno. En muchos casos, el segmento directivo en el Perú se encuentra actualmente en un estado preburocrático, es decir, ni siquiera habría pasado a ser parte de una cultura regida por el profesionalismo de 
sus miembros, sino más bien por la fidelidad al político de turno (SERVIR 2015).

$\mathrm{Al}$ respecto, SERVIR realizó un estudio sobre cuáles eran los problemas que afrontaba el segmento directivo en el Perú de cara a la implementación del Cuerpo de Gerentes Públicos. El estudio concluyó que el problema central del segmento directivo es la insuficiencia de recursos humanos con las competencias necesarias para gestionar las entidades públicas del Estado (SERVIR: 2015). Este modelo politizado de dirección ha generado problemas endémicos en el segmento directivo del Estado, los cuales han impedido históricamente la continuidad de las políticas, el desarrollo y la competitividad en el país. Tampoco ha permitido la evaluación de resultados en beneficio de la población y la inversión pública. Estos problemas secundarios (que alimentan el problema central) son los siguientes:

- Pocas capacidades gerenciales para realizar función directiva

- Inadecuada organización, incorporación y administración de directivos.

- Función directiva poco atractiva para atraer y retener talento.

- Escasa y precaria evaluación de directivos.

- Contexto inestable vinculado a un escaso soporte político-institucional.

Mientras estos problemas no sean resueltos, se seguirá contando con una administración pública que en su generalidad puede considerarse basada en un sistema de botín o spoil system, en detrimento del desarrollo de país y sus ciudadanos y ciudadanas (SERVIR 2015).

En el contexto del sistema de la Función Directiva, se le asignó a la Autoridad Nacional del Servicio Civil, como ya se mencionó, la misión de crear un Cuerpo de Gerentes Públicos, en forma de un laboratorio o prueba piloto, en el que se pudieran incorporar profesionales con alta capacidad para ser destinados a entidades gubernamentales.

\section{¿CÓMO SE DEFINE EL CUERPO DE GERENTES PÚBLICOS Y EN QUÉ CONSISTE SU MODELO DE GESTIÓN?}

Con base en Corrales (2010b), el Cuerpo de Gerentes Públicos se puede definir como un cuerpo de profesionales altamente competente, seleccionados en concursos públicos nacionales con el fin de ser asignados a cargos directivos o de gerencias de mando medio de entidades públicas del gobierno nacional, gobiernos regionales y locales, con el propósito de mejorar la gestión pública en todos los niveles de gobierno. 
Conforme a SERVIR (2015), el diseño e implementación de su modelo de gestión responde a un modelo de carácter gerencial que se basa en la preponderancia del mérito, la creación de valor público y la gestión por competencias. Se diferencia del resto de funcionarios públicos en que disponen de un espacio de independencia para la toma de decisiones, son evaluados en función de sus resultados y gerencian bajo criterios de valor público, responsabilidad de resultados y racionalidad económica (SERVIR 2015).

Para Corrales Ángulo (2010b), se busca en el modelo de gestión una política remunerativa competitiva, con la finalidad de atraer y retener profesionales altamente capacitados; una evidencia de tal propósito es que en el Decreto N. ${ }^{\circ} 1.024$ se prevé que su remuneración podrá superar en un $30 \%$ a la retribución correspondiente a un ministro, aunque ello no se aplica. De esta forma, la remuneración de un miembro del cuerpo comprende una escala básica, una bonificación mensual por cambio de residencia habitual.

La asignación es a demanda, esto es, son las entidades públicas las que solicitan personal para determinados puestos. Conforme a Barrios (2012), los profesionales seleccionados se incorporan al Cuerpo de Gerentes Públicos y son asignados a una entidad de destino, en principio por tres años prorrogables, por lo que al término del periodo pueden ser asignados a otra posición, o en otra entidad pública que también haya requerido la asignación de gerentes públicos. La coordinación de las actividades de desarrollo del modelo de gestión está a cargo de SERVIR. Consta de los siguientes procesos:

- Selección. El proceso para ingresar se realiza a través de un concurso público nacional conducido por SERVIR y consiste en dos etapas. Corrales Ángulo (2010b) refiere que la primera etapa puede ser realizada total o parcialmente por una empresa terciarizada ${ }^{4}$, que se encarga del reclutamiento a nivel nacional y de una preselección de candidatos. Originalmente, a través de la intervención de la empresa privada se busca otorgar mayor legitimidad a los nuevos procesos y aprovechar el expertise de organizaciones especializadas en la selección de ejecutivos por competencias. Posteriormente SERVIR desarrolló ese expertise y lleva a cabo directamente esta primera etapa.

- Ingreso. El ingreso considera las siguientes etapas: 1) reclutamiento; 2) evaluación curricular a profundidad; 3) evaluación masiva; 4) entrevista por competencias; 5) verificación de referencias; y 6)

4 A partir de la quinta convocatoria, SERVIR realiza también la primera etapa. 
información de ternas (Corrales Angulo y Martín 2012). Corrales Ángulo (2010b) señala que los aspirantes aprobados pasan a la segunda etapa, en la que participan de un Curso de Introducción orientado a desarrollar una visión común del rol de gerentes públicos, alinear conocimientos claves, consolidar y evaluar competencias; este no se trata de un curso típico ya que es la última etapa del proceso de selección. Los candidatos que aprueban el curso se incorporan al Cuerpo de Gerentes Públicos. El postulante que obtiene mayor puntaje y adecuación es asignado a la posición, los restantes quedan en disponibilidad para ser asignados a otro cargo en función de nuevos requerimientos. Los candidatos deben ajustarse a un perfil tipo conformado por competencias genéricas.

- Formación. Una vez que los nuevos integrantes ingresan al cuerpo, se les incorpora a un proceso de formación continua que incluye un programa de capacitación para el desarrollo de capacidades tanto de ellos como de sus equipos de colaboradores, reuniones periódicas con gerentes públicos para compartir experiencias, acceso a un staff de expertos para consulta en diversas materias, y un programa de coaching ejecutivo impartido por una empresa especializada. Se busca el desarrollo de gerentes públicos generalistas, es decir, directivos con un conocimiento amplio y general de la gestión pública, en el que se privilegia la formación de competencias para la realización de funciones directivas (Corrales Angulo 2010b, SERVIR 2015).

- Evaluación del desempeño. Abarca dos procesos: 1) la evaluación del cumplimiento de metas definidas en el marco de los convenios suscritos entre la entidad solicitante y SERVIR; 2) la evaluación de competencias. Se considera que el gerente que no logre cumplir con el 50\% de las metas en dos evaluaciones anuales, se le excluya del Cuerpo (Corrales Angulo 2010b, Iacoviello 2011). De igual manera, Barrios (2012) destaca que en el Decreto Legislativo N. ${ }^{\circ} 1.024$ se establece un enfoque de evaluación por resultados, consistente en que la entidad solicitante y la autoridad firman un convenio en el que se definen las funciones y las responsabilidades del cargo, las metas que se espera del desempeño del gerente público y los indicadores cuantificables para su evaluación.

- Permanencia. Si bien el gerente público goza de estabilidad en el puesto por el periodo de convenio de asignación, la permanencia depende del desempeño satisfactorio, del carácter transitorio del servicio y de la demanda de las entidades de destino sobre el pool de gerentes públicos disponibles. Además está sujeto a ser removido por la entidad de destino por causas relacionadas a la comisión de una falta disciplinaria o por rendimiento deficiente. (Corrales Angulo 2010b, Decreto Legislativo N. ${ }^{\circ}$ 1.024). 
- Disponibilidad. Una vez concluida la asignación, el gerente público se posiciona en una situación de disponibilidad para su recolocación, si transcurridos dos meses la autoridad no lo logra reubicar, el gerente pasa al régimen de disponibilidad sin remuneración. Por lo que si después de doce meses consecutivos no se le logra recolocar, opera la baja automática (Corrales Angulo 2010b, Reglamento del Régimen Laboral de los Gerentes Públicos 2009).

\section{¿CUÁLES SON SUS PERFILES DE COMPETENCIAS?}

En un primer momento, SERVIR instaura un perfil genérico conformado por doce competencias, divididas en seis habilidades y seis actitudes.

\section{Tabla 1: Perfil genérico de doce competencias}

Habilidades

Liderazgo catalizador

Capacidad de gestión

Prospectiva

Sentido de urgencia

Diálogo efecctivo y articulador

Sentido común
Actitudes

\section{Confianza en sí mismo}

Relación con su entorno

Tolerancia y flexibilidad

Orientación a resultados

Honestidad y vocacipon de servicio

Compromiso institucional

Fuente: Corrales Angulo (2010b: 12-13).

Al mismo tiempo, se elaboran 18 perfiles específicos por competencias por cada uno de los puestos tipo (Corrales 2010b).De acuerdo con la Consultora Governa (2012), se define un perfil común y perfiles específicos por puestos tipo, que permite utilizar criterios similares en la selección, ya que el proceso de selección busca que el Cuerpo de Gerentes Públicos se caracterice como un sistema meritocrático en el que se priorice la capacidad, habilidades y valores de los seleccionados. Según la Consultora Governa (2012), el proceso comprende la selección de puestos tipo, esto es, los postulantes no aplican a un puesto específico sino a un puesto tipo. Para el proceso de selección se considera la información de la entidad de destino sobre el puesto a cubrir, que permita elegir entre los postulantes que aplican, al que se ajuste mejor a los requerimientos de la posición. En el armado de las ternas se procura concordar a un candidato que reúna el perfil genérico del puesto tipo, con las condiciones para el puesto específico. 
La selección de los gerentes públicos la realiza SERVIR, fija las competencias genéricas y el grado requerido de las mismas (SERVIR 2015). En un segundo momento, SERVIR establece una nueva actualización de siete competencias genéricas aplicables a los gerentes públicos. Ellas son: 1) orientación al resultado; 2) innovación y mejora continua; 3 ) comunicación efectiva; 4) liderazgo; 5) actitud de servicio; 6) sentido de urgencia; 7) trabajo en equipo (SERVIR (2015: 29).

\section{¿QUÉ RESULTADOS SE HAN OBTENIDO?}

SERVIR contrató en 2012 los servicios de la consultora privada Governa para realizar una evaluación independiente: titulada Evaluación del Diseño, Procesos y Resultados del Cuerpo de Gerentes Públicos. Entre algunos de sus resultados destaca que el número de entidades atendidas por el Cuerpo de Gerentes Públicos de SERVIR, ha ido en aumento por cada trimestre con un incremento mayor en el gobierno nacional y un pequeño aumento en los gobiernos regionales y los gobiernos locales en el periodo de 2009 a 2012 (Governa 2012).

\section{Gráfico 3: Número de entidades atendidas por el Cuerpo de Gerentes Públicos de SERVIR}

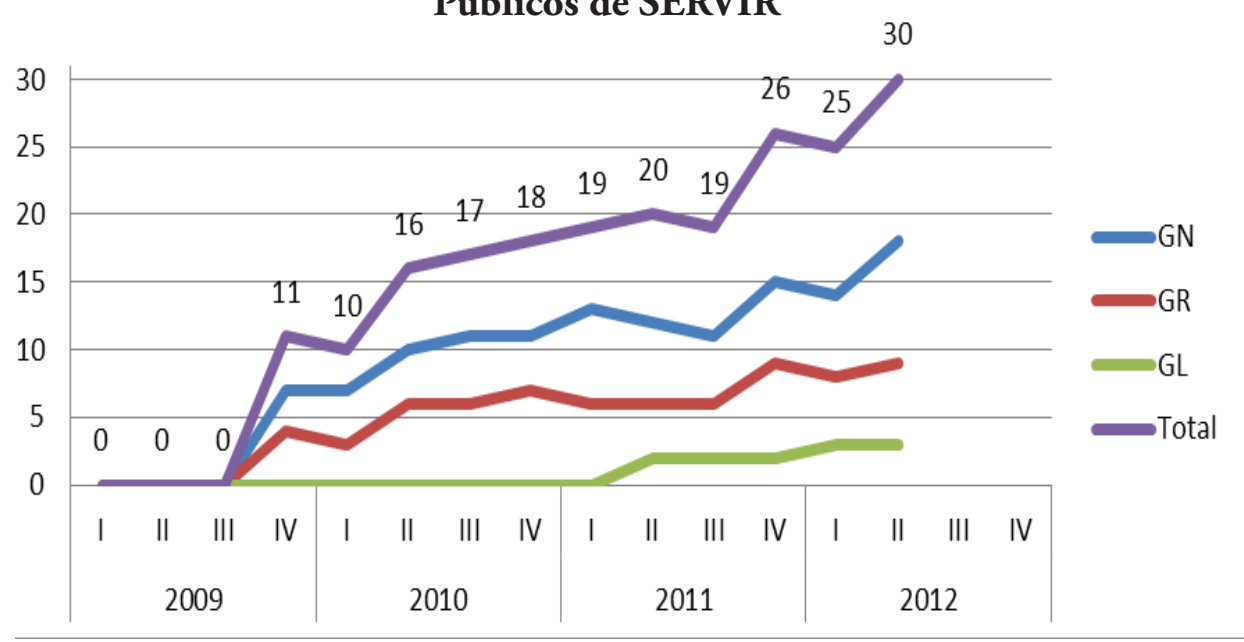

GN: Gobierno nacional; GR: Gobierno regional: GL: Gobierno local. Fuente: Governa (2012: 34).

Por otra parte, el número de Gerentes Públicos se ha incrementado a nivel nacional, con una disminución en los gobiernos regionales y un relativo aumento a nivel local. 
Gráfico 4: Número de gerentes públicos en el Gobierno de Perú 30

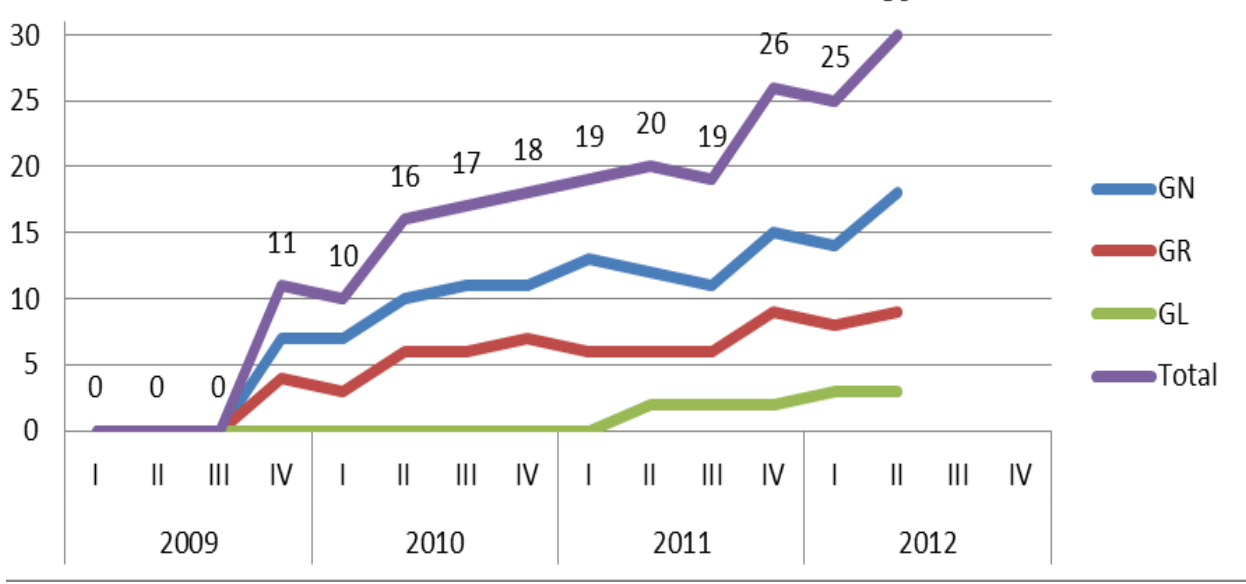

GN: Gobierno nacional; GR: Gobierno regional: GL: Gobierno local. Fuente: Governa (2012: 34).

Como parte de la consultoría, Governa (2012) realizó una encuesta a los gerentes públicos que tenían más de un año de asignados en una entidad, en la misma se les pidió identificar cuáles han sido los logros más importantes en su trayectoria como gerente público. Destacan el aporte al personal bajo el modelo de trabajo en equipo, su contribución a la adecuada ejecución del presupuesto y sacar adelante mejoras en procesos. 


\section{Gráfico 5: Logros más importantes en su trayectoria como gerente} público

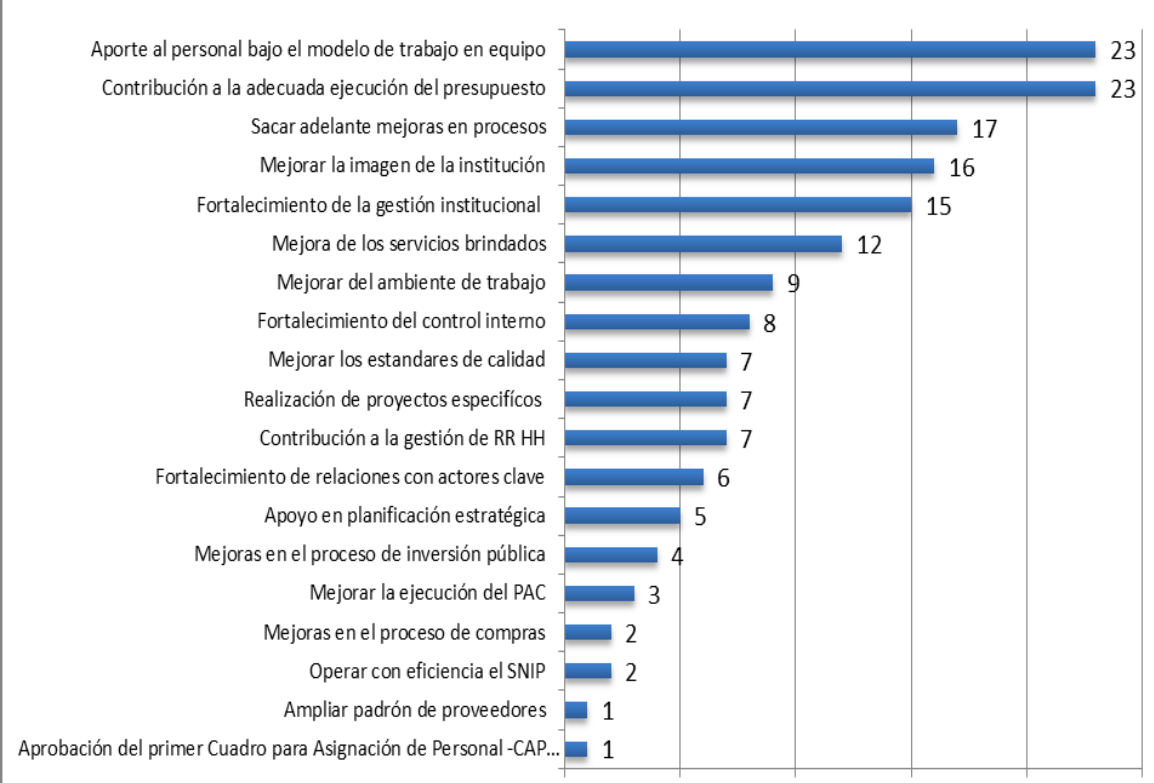

Fuente: Governa (2012: 38).

Mientras que entre los principales problemas que enfrentaban los gerentes públicos para desarrollar su labor figuraron: falta de recursos humanos calificados, limitación de personal, carencia de recursos financieros y de tecnologías de información.

\section{Gráfico 6: Principales problemas de los gerentes públicos para desarrollar su labor}

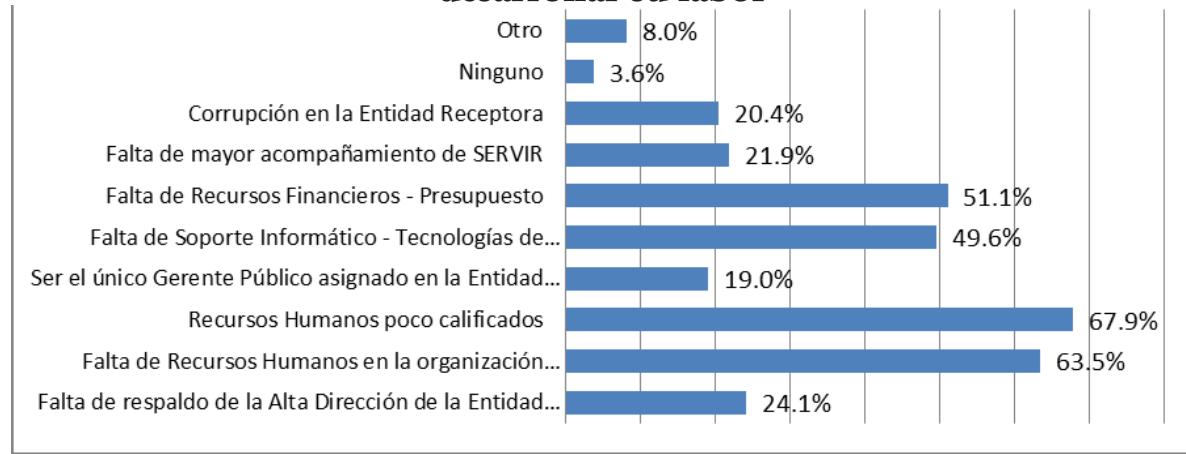

Fuente: Governa (2012: 32).

Por último, resalta que, al consultar sobre la satisfacción de los jefes con los gerentes públicos, el $56 \%$ se encontraba muy satisfecho, el $42 \%$ satisfecho y solo un $2 \%$ insatisfecho. 


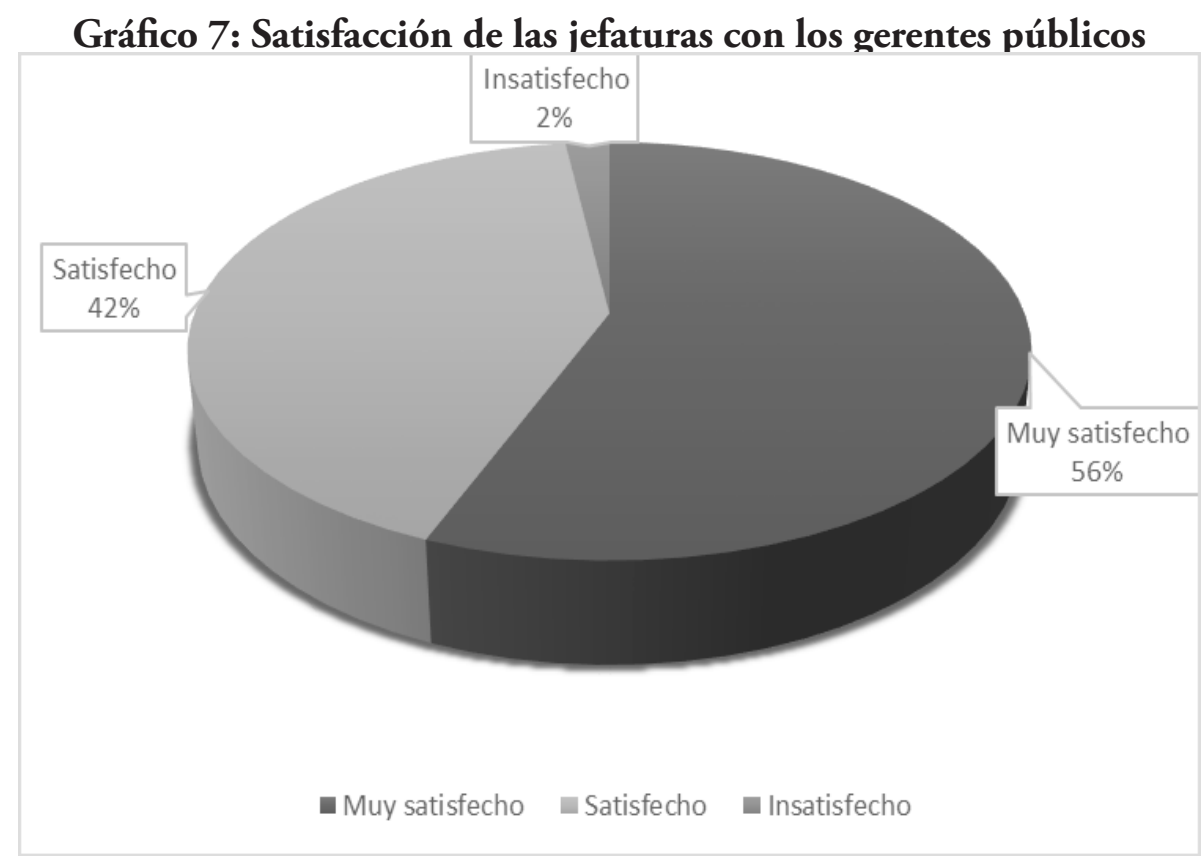

Fuente: Governa (2012: 45).

\section{¿QUÉ FORTALEZAS Y ÁREAS DE OPORTUNIDAD SE OBSERVAN?}

Para este apartado, centraremos nuestra atención en el cuerpo de gerentes públicos, en el que identificamos una serie de fortalezas y debilidades que se describen a continuación.

\section{ForTalezas}

- Los procesos de selección son abiertos a todos los candidatos que reúnen los requisitos provenientes de los sectores público o privado ${ }^{5}$ (SERVIR 2015). Por lo tanto, no se ejerce una discriminación para gente proveniente del sector privado. Tratándose sobre todo de puestos cuya experiencia previa en el sector gubernamental es fundamental en algunos sentidos.

- Dispone de funcionarios públicos profesionalizados, en los que

5 En el caso del Cuerpo de Gerentes Públicos, tradicionalmente se ha requerido experiencia previa en el Estado, de forma directa o indirecta, aunque podían presentarse postulantes que estuvieran en el sector privado, siempre que tuvieran dicho requisito; recientemente se ha flexibilizado el mismo. Respecto de los directivos públicos de la Ley del Servicio Civil, para los puestos de línea no se estaría exigiendo experiencia previa en el Estado. 
se conjuga la formación técnica con la formación política y se constituyen -o al menos esa es la intención- en una bisagra entre la clase política y los servidores públicos.

- Contribuye a mejorar los aspectos de gestión de las entidades públicas, dado los procesos de reclutamiento y selección a los que se someten previamente (Governa 2012).

- Al establecer una temporalidad en el contrato (tres años) pero sujeta a su renovación, da las posibilidades que el directivo público haga una carrera en la entidad en la que se encuentra.

- Se observa que se fortalece la cooperación y ayuda mutua entre los gerentes públicos, ubicados en las diferentes entidades públicas de los distintos niveles de gobierno, lo que puede dar lugar a la gestión del conocimiento y al archivo de buenas prácticas.

- La disponibilidad de directivos generalistas favorece su movilidad en diversas entidades públicas y facilita la articulación entre las organizaciones (SERVIR 2012).

- El hecho de que funcione como un laboratorio, permite probar instrumentos de gestión de recursos humanos, analizar su viabilidad y servir de base para la adopción o no de las mismas en la administración pública (Governa 2012).

- Se propicia la rendición de cuentas y el desempeńo creciente, a partir del establecimiento de metas con indicadores por alcanzar, para cada directivo. Lo que, incluso, lleva a que se promueva una competencia entre los mismos.

\section{ÁREAS DE OPORTUNIDAD}

- Se considera que la existencia de dos sistemas de profesionalización de directivos no es adecuado, ya que da lugar a pensar y a socializar la idea de que un sistema es bueno con respecto a otro. Eso no contribuye a la construcción de una cultura solida al respecto. Lo deseable, así sea un laboratorio, es que exista únicamente uno.

- El hecho de que el sistema de función directiva, constituido por el Cuerpo de Gerentes Públicos, sea un laboratorio o prueba piloto, no implica obligaciones para las entidades, se observa que es potestativo para la entidad que quiera apoyarse en él. Por lo que, paralelamente se sigue utilizando el sistema que da lugar a la discrecionalidad y confianza políticas para ocupar los puestos directivos de las entidades.

- Su calidad de laboratorio, le otorga un elevado nivel de fragilidad y vulnerabilidad en cuanto a su posible extinción en un cambio de gobierno.

- Por lo visto, proceso de selección y el tiempo que toma el mismo, es criticada por las entidades demandantes (Governa 2012), Por lo que 
se requiere trabajar en su simplificación ${ }^{6}$.

- La conveniencia de identificar y clarificar muy bien las causales en cuanto a salida de directivos por diferencias (no necesariamente técnicas) con los políticos en turno. El porcentaje de un estimado de $30 \%$ nos parece muy elevado. Según cifra otorgada por el Gerente de Desarrollo de la Gerencia Pública de SERVIR, Andrés Corrales (entrevista realizada por el autor, 10-07-15).

- El sistema todavía no es atractivo en la oferta salarial para captar a los niveles de profesionales más especializados (Governa 2012).

- La aprobación de un régimen paralelo (Ley N. ${ }^{\circ} 29.806^{7}$ ) con características similares, pero con mayor discrecionalidad que una vía meritocrática, llama la atención respecto a la viabilidad de reducir la participación de la confianza política en la administración pública (Governa 2012).

- Algunas áreas, tanto sustantivas como adjetivas, seguramente por el sistema de botín prevaleciente, son insuficientes y poco calificadas en algunas entidades, lo que dificulta el trabajo de los Gerentes Públicos. Aquí el problema es al revés, directivos muy bien calificados, dada la forma en que ingresaron y los formaron con equipos débiles.

- El reto de expandir y profundizar el esfuerzo profesionalizador de esta Iniciativa hacia todos los demás directivos, en el menos tiempo posible, para que el esfuerzo técnico coincida con los tiempos políticos.

\section{CONCLUSIONES}

En lo que va de 2016, concretamente en el primer semestre, se han registrado elecciones en el Perú, y se ha consolidado la llegada de Pedro Pablo Kuczynski por el Partido Peruanos por el Kambio (PPK), lo que significa entre muchas cosas, y más aún en el marco de lo que acostumbran hacer muchos gobiernos de la región latinoamericana (inclusive, tratándose del mismo partido), un proceso inminente de muchos cambios. Ellos van desde la desaparición o incorporación de organizaciones (ministerios, empresas públicas) y en la misma medida de políticas públicas, hasta lo

6 Para poder tener un tiempo de respuesta más pronto para los requerimientos de las entidades, actualmente los procesos de selección de los gerentes públicos se realizan no para puestos específicos sino para puestos tipo, de modo que los requerimientos se atienden con gerentes públicos ya seleccionados que se encuentran en situación de disponibilidad.

7 Ley N. ${ }^{\circ}$ 29.806, publicada en 2011, regula la contratación de personal altamente calificado, sin enfatizar en la meritocracia como principal condición para acceder al sector público. 
más común en el que el cambio es casi total, sobre todo de titulares en las diferentes carteras de la administración pública. Esto, seguramente, derivado de cambios en la agenda político-administrativa que se establezca. Y, obviamente, la experiencia del programa de gerentes públicos no será la excepción. Sin duda alguna, se prevé que este programa, tanto como organización y política, vivirá una verdadera prueba de fuego en los próximos meses de lo que resta del ańo.

En ese sentido, nos parece que se tienen que conjugar una serie de factores para su sobrevivencia, que van desde mostrar resultados positivos, la puesta en marcha de un ejercicio profundo de negociación en el que se hagan valer los pro y contra de una política de esta envergadura, así como dar a conocer qué se espera de un proyecto como este para el futuro de ese país. Bajo esa tesitura, nos parece que se tiene que conseguir el apoyo desde el más alto nivel tanto de autoridades ejecutivas como legislativas, para que entre en vigencia esta política y, con base en la ley que lo sustenta, adquiera el piso necesario para su continuidad y, por lo tanto, deje de ser una prueba piloto o laboratorio.

Una de las ventajas que se observa de esta política de la función directiva, es que en ella se han trabajado ya varios años, aunado a la existencia de una inversión económica importante. Esto es no menor, ya que si esta política se hubiera establecido de manera reciente, despertaría la sospecha de que la pretensión es más de captura de las altas posiciones que de profesionalización, tal como ha sucedido con otras experiencias en la región a través de leyes o normas que se aprueban al final de los períodos de gobierno.

En fin, creemos que lo que se viene para esta política que busca el fortalecimiento de la función directiva peruana, es un período de valoraciones en cuanto a su continuidad o no, y de valoraciones respecto a su importancia hoy día en el funcionamiento administrativo y político del Perú. Se insiste en que es una buena propuesta, más allá de los cambios y ajustes que requiere, por lo que desde esta mirada, se hacen votos para que prevalezca y, si no es así, se haga desde una valoración adecuada y no solo desde la conveniencia político-partidista en detrimento de beneficios a la propia administración y a la ciudadanía. 


\section{REFERENCIAS}

Alza Barco, C. (2012). Documento Final. Producto 3. Consultoría para la Elaboración del Estudio sobre la Evolución de la Politica del Servicio Civil Peruano durante los últimos 30 años (1980-2011) para la Autoridad Nacional del Servicio Civil- SERVIR. Lima: SERVIR.

Banco Interamericano de Desarrollo (BID). (2013). Al servicio del Ciudadano: Una Década de Reformas del Servicio Civilen América Latina (2004-2013). Washington: Banco Interamericano de Desarrollo.

Barrios Ipenza, E. (2012). ¿Tecnócratas, Politécnicos o Tecnopols? Una Aproximación al Perfil de los Directivos Públicos del Cuerpo de Gerentes Públicos. Lima: Universidad Continental.

Brosnahan, J. (2000). Public Sector Reform Requires Leadership. En Organización para la Cooperación y el Desarrollo Económicos (OCDE) (Ed.), Government of the Future. Paris: OCDE.

Centro Latinoamericano de Administración para el Desarrollo (CLAD) y Naciones Unidas (UN). (2004). Carta Iberoamericana de la Función Pública. Revista Servicio Profesional de Carrera, I (2).

Corrales, A. (2010a). La Influencia de la Alta Dirección Pública de Chile en el Cuerpo de Gerentes Públicos de Perú. Ponencia presentada en el XV Congreso Internacional del CLAD sobre la Reforma del Estado y la Administración Pública, 9-12 de noviembre, Santo Domingo, República Dominicana,

(2010b). Diseño e implementación del Cuerpo de Gerentes Públicos en Perú. Ponencia presentada en el XV Congreso Internacional del CLAD sobre la Reforma del Estado y la Administración Pública, 9-12 de noviembre, Santo Domingo, República Dominicana,

Corrales Angulo, A. y Martín, A. (2012). Aportes del Cuerpo de Gerentes Públicos a la Reforma del Servicio Civil en el Perú. Ponencia presentada en el XVII Congreso Internacional del CLAD sobre la Reforma del Estado y de la Administración Pública. 30 de octubre al 2 de noviembre, Cartagena, Colombia.

Dauphinais, W. y Price, C. (1988). Straight from the CEO. Nueva York: Price Waterhouse. 
Figueroa, R. (2002). Regímenes de Directivos Públicos en América Latina: Estado de Arte, Análisis y Conclusiones. Washington: Banco Interamericano de Desarrollo.

Gardner, J. (1990). On Leadership. Nueva York: The Free Press.

Governa S. A. C. (2012). Consultoría para la Evaluación del Diseño, Procesos y Resultados del Cuerpo de Gerentes Públicos. Informe Final. Lima: Governa. Heifetz, R. (1994). Leadership without Easy Answers. Cambridge: Harvard University Press.

Iacoviello, M. (2011). Diagnóstico de Servicio Civil de Perú. Informe Final. Washington: Banco Interamericano de Desarrollo.

Kliksberg, B, (1983). Universidad, Formación de Administradores y Sector Público en América Latina. México: Fondo de Cultura Económica, INAP, CLAD.

Kotter, J. (1983). Lo que Hacen Realmente los Buenos Directores Generales. Harvard-Deusto Business Review, 15, 55-68.

Laufer, R-y Burluad, A. (1989). Dirección Pública: Gestión y Legitimidad. Madrid: INAP.

Likert, R. (1961). New Patterns of Management. Nueva York: Mc Graw Hill.

Lodge, M. y Wegrich, K. (2014). The Governance Report 2014. Oxford: Oxford University Press.

Martínez Puón, R. (2011). Directivos versus Políticos. La Importancia de la Función Directiva en las Administraciones Públicas. México: Miguel Ángel Porrúa.

Mintzberg, H. (1983). La Naturaleza del Trabajo Directivo. Barcelona: Ariel.

(1996). Managing Government, Government Management. Harvard Business Review, mayo-junio, 75-83.

. (2005). Directivos, No MBAs. Una Visión Critica de la Dirección de Empresas y la Formación Empresarial. Barcelona: Deusto. 
. (2002). Developing Leaders? Developing Countries?

Development in Practice, 16 (1), 4-14.

Organización para la Cooperación y el Desarrollo Económicos (OCDE). (1999). The State of the Higher Civil Service After Reform: Britain, Canada and the United States. Paris: OCDE.

. (2008). The Senior Civil Service in National Governments of OECD Countries. Paris: OCDE.

Sayles, L. R. (1964). Managerial Behavior: Administration in Complex Organizations. Nueva York: Mc Graw Hill.

SERVIR. (2015). Documento de Trabajo No 1-2015. Marco Conceptual del Grupo de Directivos Públicos del Servicio Civil Peruano. Lima: SERVIR.

\section{Decretos y leyes}

Decreto Legislativo N. ${ }^{\circ} 1.023$ que crea la Autoridad Nacional del Servicio Civil, Rectora del Sistema Administrativo de Gestión de Recursos Humanos. Diario Oficial El Peruano. Normas Legales. 21 de junio de 2008, Lima, Perú.

Decreto Legislativo N. ${ }^{\circ} 1.024$ que crea y regula el Cuerpo de Gerentes Públicos. Diario Oficial El Peruano. Normas Legales. 21 de junio de 2008, Lima, Perú.

Reglamento del Régimen Laboral de los Gerentes Públicos creado por el Decreto Legislativo N. ${ }^{\circ}$ 1.024. (2009). Diario Oficial El Peruano. Normas Legales. 17 de mayo de 2009, Lima, Perú.

Ley N. 29.806, Ley que regula la contratación de personal altamente calificado en el Sector Público y dicta otras disposiciones. Diario Oficial El Peruano. Normas Legales. 24 de noviembre de 2011, Lima, Perú.

Ley N. 30.057 , Ley del Servicio Civil. Diario Oficial El Peruano. Normas Legales. 4 de julio de 2013, Lima, Perú.

Recibido: 23-05-2016

Aceptación de la versión final: 22-06-2016 